\title{
The Plant-Derived Chalcone 2,2',5'-Trihydroxychalcone Provides Neuroprotection against Toll-Like Receptor 4 Triggered Inflammation in Microglia
}

\author{
Manasi Jiwrajka, ${ }^{1}$ Alexandra Phillips, ${ }^{2}$ Matt Butler, ${ }^{2}$ \\ Miriam Rossi, ${ }^{3}$ and Jennifer M. Pocock ${ }^{2}$ \\ ${ }^{1}$ Mayne Medical School, 288 Herston Road, Brisbane, QLD 4006, Australia \\ ${ }^{2}$ Department of Neuroinflammation, University College London Institute of Neurology, 1 Wakefield Street, London WC1N 1PK, UK \\ ${ }^{3}$ Department of Chemistry, Vassar College, 124 Raymond Avenue, Poughkeepsie, NY 12604-0484, USA
}

Correspondence should be addressed to Jennifer M. Pocock; j.pocock@ucl.ac.uk

Received 20 July 2015; Accepted 30 September 2015

Academic Editor: Felipe Dal Pizzol

Copyright (C) 2016 Manasi Jiwrajka et al. This is an open access article distributed under the Creative Commons Attribution License, which permits unrestricted use, distribution, and reproduction in any medium, provided the original work is properly cited.

\begin{abstract}
Chalcones are plant metabolites with potential for therapeutic exploitation as antioxidant, anti-inflammatory, and antiproliferative agents. Here we explored the neuroprotective effects of $2,2^{\prime}, 5^{\prime}$-trihydroxychalcone (225THC), a potent antioxidant with radicalscavenging properties. 225THC was found to be a potent inhibitor of apoptosis in stimulated primary rat neuronal cultures. This was likely mediated by an anti-inflammatory effect on microglial cells since 225THC inhibited LPS-stimulated TNF- $\alpha$ and IL- 6 secretion from primary rat microglia and modulated the cytokine/chemokine profile of BV2 microglial cells. Additionally, 225THC inhibited LPS-evoked inducible nitric oxide synthase expression but did not influence endogenous superoxide generation. Microglial flow cytometric analyses indicated the 225THC treatment induced a shift from an M1-like phenotype to a more downregulated microglial profile. Taken together these data suggest that the chalcone $2,2^{\prime}, 5^{\prime}$-trihydroxychalcone can modulate neuroinflammatory activation in brain-derived microglia and holds promise as a therapeutic in neuroinflammatory conditions.
\end{abstract}

\section{Introduction}

Plants produce secondary metabolites that protect them from toxins and insects. Some of these plant metabolites, such as chalcones, have significant antioxidant, anti-inflammatory, and antiproliferative properties in a range of cell types [17]. Chalcones are similar to other known antioxidants such as resveratrol, curcumin, and ubiquinone and are the natural precursors of flavonoids and isoflavonoids in higher plants $[4,8-10]$. In plants, chalcones protect against UV exposure, pathogens, and insects, and their antioxidant and antiinflammatory properties make them of increasing interest in the treatment of human conditions such as cancer, inflammation, tuberculosis, and malaria $[2,7,11]$.

Stress and injury to cells can cause the production of free radicals and the release of cytokines. In the brain, such substances are produced by the activation of microglia, the brain's resident phagocytes, leading to neurotoxicity [12-14]. During ageing, neurodegeneration, ischaemia, brain injury, or other neuropathologies, there is enhanced production of free radicals and cytokines, increased apoptosis, and reduced expression of synaptic or growth proteins [15-18].

In the brain, the chalcone isoliquiritigenin has anxiolytic effects [19] whilst two chalconoids from the desert plant Pulicaria incisa inhibited the production of reactive oxygen species (ROS) by astrocytes and prevented their oxidantinduced cell death [20]. One plant-derived chalcone, 2,2, $5^{\prime}$ trihydroxychalcone (225THC), demonstrated strong antioxidant and radical-scavenging properties in L-6 myoblasts and THP-1 human monocytes [21]. However, the neuroprotective effects of this particular chalcone on cells of the CNS are unknown and the subject of the present study. 


\section{Materials and Methods}

\subsection{Cell Culture}

2.1.1. BV2 Microglia. The BV2 mouse microglial cell line was a kind gift from Dr. Claudie Hooper, Institute of Psychiatry, Kings College London, and was originally obtained from the Department of Life Sciences, National Cheng Kung University, Taiwan. The cells were cultured in RPMI-1640 medium (Gibco, Life Technologies) plus 5\% foetal bovine serum (FBS) and $50 \mathrm{U} / \mathrm{mL}$ penicillin and $50 \mu \mathrm{g} / \mathrm{mL}$ streptomycin (all Invitrogen, http://www.invitrogen.com/) at $37^{\circ} \mathrm{C}$, at $5 \% \mathrm{CO}_{2}$, and at a density of $2 \times 10^{4}$ per $13 \mathrm{~mm}$ glass coverslips in 24-well plates or $1 \times 10^{5}$ per 6-well plate for Western blotting or FACS analyses and rested overnight at $37^{\circ} \mathrm{C}$ plus $5 \% \mathrm{CO}_{2}$. Before use, cell medium was replaced with RPMI plus $1 \%$ FBS.

\subsubsection{Primary Cultures of Microglia and Cerebellar Granule} Cell Neurons. Sprague Dawley rat pups (postnatal day 5) were bred and reared in-house from stock animals (Charles River UK, Kent, UK) and were sacrificed in accordance with Schedule 1 of the Animals Scientific Procedures Act (1986) UK, for the culture of primary microglia and cerebellar neurons. Microglia were cultured as described previously [22]. Briefly, cells were plated on $13 \mathrm{~mm}$ glass coverslips in 24 well plates at $0.5 \times 10^{5}$ cells/well and maintained in culture medium consisting of MEM (Invitrogen, http://www.invitrogen.com/), supplemented with $10 \%$ FBS, $20 \mathrm{mM} \mathrm{KCl}, 20 \mathrm{mM}$ D-glucose, $2 \mathrm{mM} \mathrm{D}$-glucose, $25 \mathrm{mM}$ $\mathrm{NaHCO}_{4}, 50 \mathrm{U} / \mathrm{mL}$ penicillin, $50 \mu \mathrm{g} / \mathrm{mL}$ streptomycin, and $6 \mu \mathrm{g} / \mathrm{mL}$ ampicillin. The microglia were maintained at $37^{\circ} \mathrm{C}$, $6 \% \mathrm{CO}_{2}$ and used 1 day after plating. Where possible experiments were carried out using primary microglia but where assays required higher cell numbers, BV2 microglia were used. We have previously compared responses of BV2 microglia and primary microglia and have found not obvious differences in responses. Cerebellar granule cells (CGCs) were cultured as described previously [22]. The cells were plated on $13 \mathrm{~mm}$ glass coverslips in 24 well plates at a density of $8 \times 10^{5} /$ coverslip and maintained in culture medium (MEM plus 10\% FBS, $20 \mathrm{mM} \mathrm{KCl,} 30 \mathrm{mM} \mathrm{D}$-glucose, $2 \mathrm{mM}$ L-glutamine, $25 \mathrm{mM} \mathrm{NaHCO}$, $50 \mathrm{U} / \mathrm{mL}$ penicillin, $50 \mu \mathrm{M}$ streptomycin, and $6 \mu \mathrm{g} / \mathrm{mL}$ ampicillin) that was later supplemented with $20 \mu \mathrm{M}$ cytosine furanoarabinoside (Ara-C) to control glial proliferation. The cells were maintained at $37^{\circ} \mathrm{C}$, $6 \% \mathrm{CO}_{2}$ and used after 8 days in vitro (8 DIV).

2.2. Chalcone Treatment. The chalcone $2,2^{\prime}, 5^{\prime}$-trihydroxychalcone (225THC) was purchased from Indofine Chemical Co. (Hillsborough, NJ, USA, at 97\% purity) and was applied to microglia and neurons to test for any inherent toxicity. $225 \mathrm{THC}$ was added at (final concentrations) $1 \mu \mathrm{M}, 5 \mu \mathrm{M}$, $10 \mu \mathrm{M}, 25 \mu \mathrm{M}, 50 \mu \mathrm{M}, 100 \mu \mathrm{M}$, or $500 \mu \mathrm{M}$ in $100 \%$ sterile DMSO (Sigma D2650; hybri-MAX) as well as a solvent control of $100 \%$ DMSO and incubated for $24 \mathrm{~h}$. Live/dead assays were carried out using Hoechst 33342 for total cell number and propidium iodide (PI) for dead cells as previously described [22]. Cells were visualised and images captured with a Zeiss Axioskop 2 fluorescence microscope
(Oberkochen, Germany) and images were captured using Zeiss Axiovision Imaging System 4.8 software. The number of live/dead cells was counted manually, or using Image J software. At least 5 fields per coverslip, 3 coverslips per 225THC concentration from 3 separate cell platings were analysed.

2.3. 225THC Treatment of Surveillant and Inflammatory Microglia. The effects of 225THC on microglial activation were tested by treating primary microglia or BV2 microglia with $225 \mathrm{THC}(1 \mu \mathrm{M}, 5 \mu \mathrm{M}, 10 \mu \mathrm{M}, 50 \mu \mathrm{M}, 100 \mu \mathrm{M}$, and $500 \mu \mathrm{M}$ ) with or without $2 \mu \mathrm{g} / \mathrm{mL}$ of the Toll-like receptor 4 (TLR4) agonist, lipopolysaccharide (LPS), for $24-48 \mathrm{~h}$. This concentration of LPS was used as this produces maximal iNOS expression as determined in previous experiments and published papers from our laboratory $[14,23]$. The cells were subsequently analysed by live/dead assay, Western blot, and cell supernatants analysed for cytokine secretion as described. To test for protective effects of 225THC against neuroinflammation-driven neurotoxicity, CGCs were directly treated with $225 \mathrm{THC}(5 \mu \mathrm{M}, 50 \mu \mathrm{M}$, and $500 \mu \mathrm{M})$ and then either $2 \mu \mathrm{g} / \mathrm{mL}$ LPS or $10 \mathrm{ng} / \mathrm{mL}$ IFN- $\gamma$ to activate resident microglia in the cultures. Following $24 \mathrm{~h}$, CGC cultures were analysed by Hoechst 33342 staining to assess nuclear morphology as described previously [22].

2.4. Western Blot of Inducible Nitric Oxide Synthase Expression. Cells were treated for Western blotting using standard techniques followed by blot visualisation with ECL. Beta- $(\beta-)$ actin was used as a loading control in all gels and protein bands analysed following densitometry with Image J software. Primary antibodies used were anti-iNOS, 1:2500, overnight followed by 1:5000 HRP conjugated goat antirabbit, for $1 \mathrm{~h}$, and anti- $\beta$ actin $1: 10000$ overnight, followed by HRP conjugated goat anti-mouse, 1:20,0000 for $1 \mathrm{~h}$. Goat anti-rabbit peroxidase secondary antibody was from Sigma (Poole, UK), donkey anti-goat peroxidase secondary antibody was from GeneTex (Insight Biotech, Wembley UK), goat anti-arginase-1 was from Santa Cruz Biotech (http://www.scbt.com/), and rabbit anti-inducible nitric oxide synthase (iNOS) was from BD Biosciences (http://www .bdbiosciences.com/).

2.5. Dihydroethidium Fluorescence Imaging of Superoxide Generation. The superoxide sensitive fluorescent dye dihydroethidium (dHEth) was used to assess microglial superoxide generation and its regulation by the chalcone. Dihydroethidium is oxidised to 2-hydroxyethidium $\left(2-\mathrm{OH}-\mathrm{E}^{+}\right)$ upon exposure to superoxide specifically, correlating with a shift in fluorescence from blue to red which is detectable by fluorescence microscopy [24] and we have used this previously to assess superoxide generation in microglia [22]. BV2 microglia were treated with 225THC, LPS, or $10 \mathrm{nM}$ phorbol 12-myristate 13-acetate (PMA) (the latter as a positive control for the generation of superoxide by NADPH activity $[22,25])$ for $24 \mathrm{~h}$ and then incubated with $5 \mu \mathrm{M}$ dHEth for $40 \mathrm{~min}$ to identify superoxide-producing microglia as described previously [22] plus $0.6 \mu \mathrm{g} / \mathrm{mL}$ Hoechst 33342 for $40 \mathrm{~min}$ was used to counterstain all nuclei for total cell 
number. Microglia were imaged by fluorescence microscopy as above, and superoxide-positive microglia, as indicated by red nuclei, were counted and expressed as a percentage of total cell number. To ensure that the red fluorescence was due to superoxide production, experiments were carried out by incubation of the cells with $10 \mu \mathrm{M}$ apocynin [22].

2.6. ELISA of Secreted Cytokines by Microglia. TNF- $\alpha$ or IL-1 $\beta$ concentrations in primary rat microglia cell culture supernatants were quantified using Quantikine Rat TNF- $\alpha$ or IL-1 $\beta$ Immunoassay kit according to the manufacturer's instructions (R\&D Systems, Abingdon UK). Cytokine concentrations in cell supernatants were determined against a standard curve of TNF- $\alpha$ or IL-1 $\beta$. In addition, a range of inflammatory mediators were analysed in BV2 cell culture supernatants with an Inflammatory ELISA strip assay according to the manufacturer's instructions (Signosis Inc., Caltag MedSystems Ltd., Buckingham, UK). In this case, all values were presented as a percentage of control values. For each condition, the cytokine content in supernatants was analysed from three coverslips of microglia in three independent cell platings with each sample assayed in duplicate. All other chemicals and reagents were from Sigma (Poole, UK).

2.7. Flow Cytometric Analysis of Microglial Inflammatory Markers. BV2 cells were treated with 1,5 , or $10 \mu \mathrm{M}$ of 225THC for 2 hours prior to the addition of $2 \mu \mathrm{g} / \mathrm{mL}$ LPS for a further 48 hours. Cells were harvested by washing in Dulbecco's phosphate buffered saline (PBS, without $\mathrm{Ca}^{2+}$ and $\mathrm{Mg}^{2+}$ ) for 20 minutes at $37^{\circ} \mathrm{C}$ and then resuspended in cold PBS containing $0.5 \% \mathrm{BSA} / 0.05 \% \mathrm{NaN}_{3}$ to metabolically fix the cells. Cells were stained with anti-mouse CD11b-FITC, CD40-FITC, CD54-FITC, and CD68-FITC, or appropriate FITC-conjugated isotype control antibodies (Miltenyi Biotec, UK) as per the manufacturer's instructions and cell surface staining was assessed by flow cytometry (FACSCalibur running CellQuest Pro; Becton Dickinson, UK) and analysed using Flowing software v2.5.1. Data are presented as average fold change in mean fluorescence intensity (MFI) versus untreated cells \pm SEM from 4 independent experiments.

2.8. Statistical Analysis. Statistical analyses were performed using one-way ANOVA with Tukey post hoc analysis, where comparisons were made between treatments and control untreated cells, and also between treatment groups as indicated. Where stated, Student's $t$-test was used and all data were from 3 separate experiments, ${ }^{*} p<0.05,{ }^{* *} p<0.01$, and ${ }^{* * *} p<0.005$ compared with controls or as indicated in figures. Where imaging was performed, a minimum of 3 fields of view were analysed from 3 coverslips per condition. Images of fluorescent fields of cells or Western blots are representative of analysed data from 3 independent experiments. The densitometry for Westerns was performed using Image J, measuring the optical density (OD) of each band and normalising to the $\beta$-actin obtained for that particular sample. For the statistical analysis of these data, each treatment was compared with control untreated cells by ANOVA analysis, and appropriate comparisons were also made between treatment groups \pm inhibitors using Student's $t$-test.

\section{Results and Discussion}

3.1. Effects of 225THC in Primary Rat Cerebellar Granule Cell Neuronal Cultures. We initially investigated whether the $225 \mathrm{THC}$ was directly toxic to neurons by incubating primary rat cerebellar granule cell (CGC) neuronal culture neurons with concentrations of $5-500 \mu \mathrm{M} 225 \mathrm{THC}$ for $24 \mathrm{~h}$. This range of concentrations was assessed as previous studies using similar compounds have identified activity within this range $[20,26,27]$. Microscopic live/dead analyses revealed no significant toxicity in these cultures with DMSO solvent control, or with 5,50 , or $500 \mu \mathrm{M}$ 225THC (Figure 1(a)) when compared with untreated cells. We next investigated the potential neuroprotective properties of $225 \mathrm{THC}$ in these cultures (which we have shown previously contain $2-5 \%$ microglia) $[28,29]$. A range of $225 \mathrm{THC}$ concentrations were preincubated with CGCs for 1 hour, followed by stimulation with $2 \mu \mathrm{g} / \mathrm{mL}$ LPS or $10 \mathrm{ng} / \mathrm{mL}$ IFN- $\gamma$ to induce an inflammatory phenotype within the CGC cultures. After $24 \mathrm{~h}$, the CGCs were imaged with Hoechst 33342 to visualise apoptotic nuclei within the cultures. LPS or IFN- $\gamma$ significantly increased the percentage of apoptotic cells in the neuronal cultures above basal (Figure 1(b)) whilst pretreatment with $225 \mathrm{THC}$ at 50 or $500 \mu \mathrm{M}$ significantly reduced the percentage of apoptotic cells in cultures treated with LPS or IFN- $\gamma$. Since LPS and IFN- $\gamma$ are potent activators of microglia, with no known effects on cerebellar neurons and since incubation with chalcone directly to these cultures, in the absence of inflammation, did not enhance basal levels of neuronal survival, it is likely that suppression of the neuroinflammatory and neurotoxic effects seen here are due to suppression of microglial responses. Thus we determined how these effects might be mediated. One proposed mechanism for the neurotoxicity observed is that activated microglia release proinflammatory cytokines that cause cytotoxicity to the neurons $[15,30,31]$.

\subsection{THC Inhibits Proinflammatory Cytokine Secretion by} LPS-Stimulated Primary Rat Microglial Cells. As a preliminary assessment, we again investigated the potential cellular toxicity of 225THC when added to primary rat microglial cultures. We observed that, in three separate experiments, a specific concentration of $50 \mu \mathrm{M} 225 \mathrm{THC}$ appeared to induce significant cell loss from coverslips in both nonstimulated and LPS-stimulated cells, whilst concentrations above and below this did not (Figure 2(a)). This was most likely due to loss of contact rather than toxicity as there was no increase in PI staining. DMSO controls did not affect cell numbers (data not shown).

We next assessed cytokine secretion in microglial cell culture supernatants under basal (nonstimulating conditions) and following LPS stimulation $(2 \mu \mathrm{g} / \mathrm{mL})$ in the presence of 50 or $500 \mu \mathrm{M} 225 \mathrm{THC}$ (Figure 2(b)). The basal secretion of TNF- $\alpha$, IL- 6 , and IL-10 was not significantly modulated by $5 \mu \mathrm{M}$ or $500 \mu \mathrm{M}$ 225THC whilst LPS-evoked TNF- $\alpha$ and IL- 6 secretion, but not IL-10, was significantly inhibited by 


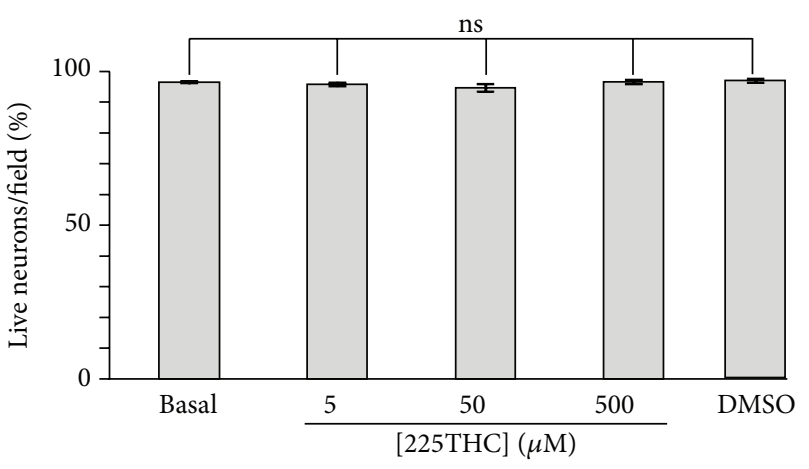

(a)

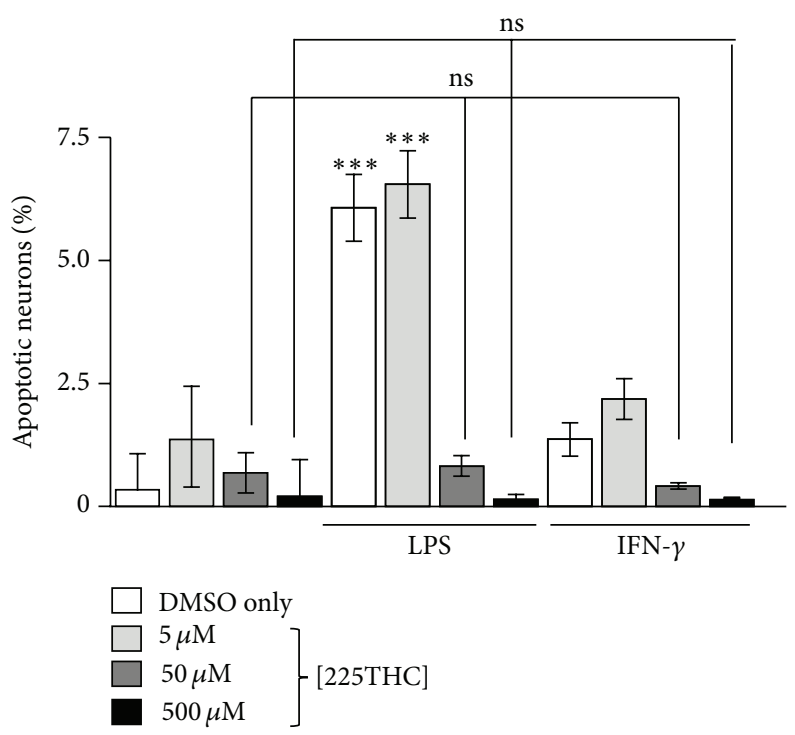

(b)

FIGURE 1: 225THC is nontoxic and neuroprotective in stimulated primary rat neuronal cultures (cerebellar granule cell neurons; CGCs). Effects of $225 \mathrm{THC}$ on primary rat CGC neurons. (a) Toxicity assay of $225 \mathrm{THC}$ on primary rat CGC neurons. Data from three separate experiments represent the mean number of live neurons (as assessed by live/dead assay) present per field of view following incubation for $24 \mathrm{~h}$ with different concentrations of $225 \mathrm{THC}(0,5,50$, and $500 \mu \mathrm{M})$ for $24 \mathrm{~h}$. DMSO is the solvent control; basal is CGCs with nothing added. Statistical analysis was performed by ANOVA with Bonferroni post hoc analysis and revealed no toxicity at the concentrations tested when all concentrations were compared with basal. The DMSO solvent control was also not toxic. (b) Direct treatment of CGC cultures for $24 \mathrm{~h}$ with 225 THC $(0,5,50$, and $500 \mu \mathrm{M})$ and $2 \mu \mathrm{g} / \mathrm{mL}$ LPS or $10 \mathrm{ng} / \mathrm{mL} \mathrm{IFN}-\gamma$ to activate resident microglia in the cultures. The percentage of apoptotic cells following Hoechst 33342 visualisation of nuclear condensation is shown for each treatment. Statistical analysis was performed by a two-way ANOVA with Bonferroni post hoc analysis; $* * *$ indicates $p<0.001$; ns, not significant when compared as indicated or with the appropriate basal control.

$5 \mu \mathrm{M}$ or $500 \mu \mathrm{M}$ 225THC (Figures 2(bi), 2(bii), and 2(biii), resp.).

\subsection{THC Modulates Inflammatory Cytokine and Chemo-} kine Responses in Murine BV2 Microglial Cells. To investigate the microglial aspects further, we used the BV2 mouse microglial cell line which is a well validated substitute for primary microglia [32]. Further examination of a lower range of $225 \mathrm{THC}$ concentrations $(1 \mu \mathrm{M}, 5 \mu \mathrm{M}$, and $10 \mu \mathrm{M})$ following a $48 \mathrm{~h}$ LPS stimulation revealed selective inhibition of LPS-induced TNF- $\alpha$, in line with our findings in primary microglia (Figure $3(\mathrm{a})$ ). In addition, the basal secretion of monocyte chemoattractant protein-1 (MCP-1) was also significantly inhibited (Figure 3(b)). We did not observe modulation of basal or LPS-evoked secretion of IFNR, IL- $1 \alpha$, IL-1 $\beta$, (data not shown) or of RANTES (Figure 3(c)) or MIP (Figure 3(d)). These data suggest that 225THC has an effect on the secretion of inflammatory cytokines such as TNF- $\alpha$, IL- 6 , and MCP-1, and the reduction of these cytokines may aid neuroprotection. Interestingly, in line with our findings, Herencia et al. [26] report that the chalcone used in their study inhibited TNF- $\alpha$ mouse air pouch tissue levels from leukocytes stimulated by zymosan, whilst Bano et al. [33] reported suppression of LPS-evoked TNF- $\alpha$ secretion from a mouse macrophage line with a synthetic chalcone. In addition, we found 225THC inhibited the secretion of two other cytokines, IL-6 and MCP-1. IL- 6 has well recognised roles in neuroinflammation and promotes microgliosis [34] whilst MCP-1 (also known as CCL-2) acts as a chemoattractant to recruit monocytes and macrophages into the brain [35]. Interestingly, recent work suggests different chalcones may selectively inhibit the secretion of different groups of cytokines and inflammatory mediators [27]. We did not see significant modulation by $225 \mathrm{THC}$ of RANTES, MIP, or IL10. Other chalcones have been shown to promote the production of neurotrophic factors such as GDNF from astrocytes [20] and this is worth future exploration for microglia.

3.4. Modulation of iNOS Expression and ROS Generation by 225THC. The expression of iNOS was assessed in BV2 microglia under basal conditions and following LPS stimulation $(2 \mu \mathrm{g} / \mathrm{mL})$ for $48 \mathrm{~h}$ in the presence of $225 \mathrm{THC}(1 \mu \mathrm{M}$, $5 \mu \mathrm{M}$, or $10 \mu \mathrm{M})$. No obvious effects were observed on the expression of iNOS in nonstimulated microglia; that is, 225THC did not induce expression of iNOS (Figure 4(a)). However LPS-evoked stimulation of iNOS expression was significantly inhibited by $225 \mathrm{THC}$ at concentrations above $5 \mu \mathrm{M}$ (Figures 4(a) and 4(b)). We next investigated microglial superoxide generation using microscopic fluorescence imaging (Figure 4(c)). No obvious effects of the 225THC were observed on the basal cell numbers expressing superoxidepositive cell nuclei and whilst the number of superoxidepositive cells was increased following LPS stimulation this was not significantly regulated by $225 \mathrm{THC}$ concentrations 


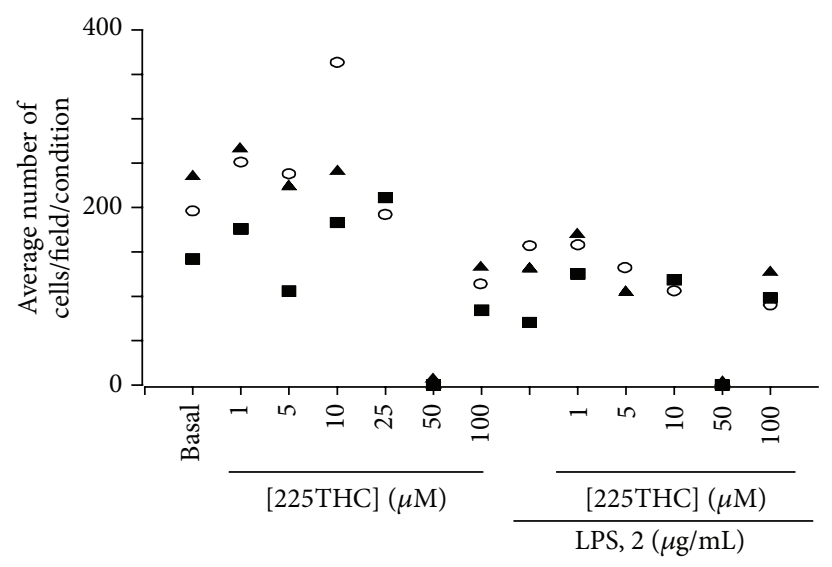

- Exp. 1

- Exp. 2

- Exp. 3

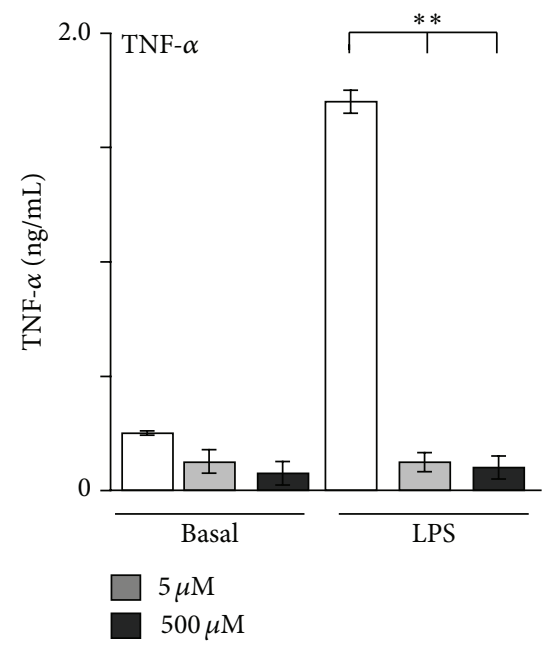

(bi)

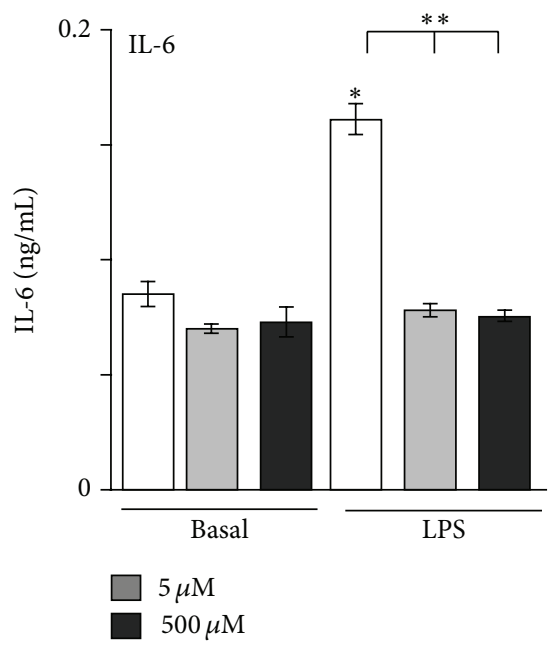

(bii)

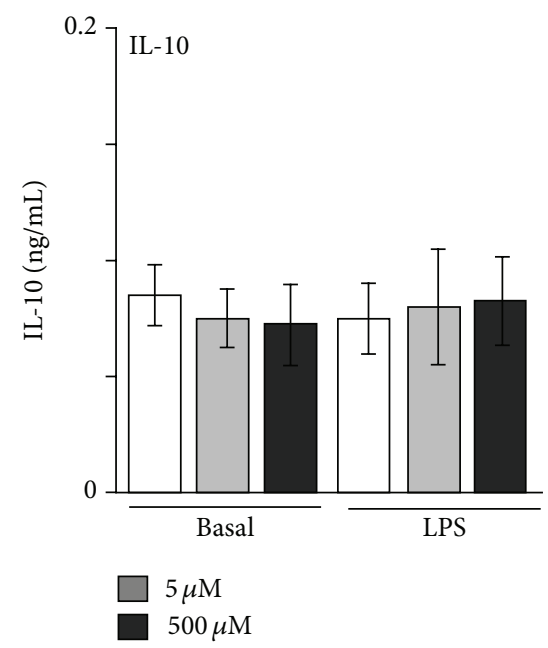

(biii)

(b)

FIGURE 2: 225THC is nontoxic and inhibits inflammatory cytokine responses in stimulated primary rat microglial cultures. Effects of 225THC on primary rat microglial cells. (a) Toxicity assay of $225 \mathrm{THC}$ on primary cultured rat microglia. Data shown from three separate experiments represent the mean number of live primary microglia (as assessed by live/dead assay) present per field of view following incubation for $24 \mathrm{~h}$ with different concentrations of $225 \mathrm{THC}(0,1,5,10,50$, and $100 \mu \mathrm{M})$ for $24 \mathrm{~h}$ with or without coactivation of the microglia with LPS $(2 \mu \mathrm{g} / \mathrm{mL})$. (b) ELISA assessment of secreted cytokines in LPS $(2 \mu \mathrm{g} / \mathrm{mL})$ activated primary rat microglia cultures treated with $225 \mathrm{THC}$ at the concentrations indicated. Statistical analysis was performed by a two-way ANOVA with Bonferroni post hoc analysis; $*$ indicates $p<0.05$, ${ }^{* *} p<0.01$; when compared with basla or as indicated.

from 1 to $100 \mu \mathrm{M}$ (Figure 4 (c)) or at $500 \mu \mathrm{M}$ (data not shown). The positive control of NADPH oxidase activation, PMA $(10 \mathrm{ng} / \mathrm{mL})$, induced a significant increase in superoxidepositive cells, in line with previous findings [22].

The inhibition of iNOS expression in microglia is in agreement with findings for another chalcone, 4-dimethylamino- $3^{\prime}, 4^{\prime}$-dimethoxychalcone, on mouse macrophages stimulated with zymosan [22]. This chalcone inhibited iNOS expression with a similar concentration $(\sim 10 \mu \mathrm{M})$ to the chalcone used in the present study. Herencia et al. [26] proposed that the effect on iNOS expression was via upstream modulation of NADPH oxidase-mediated intracellular signalling.
Since we did not detect modulation of microglial superoxide generation by $225 \mathrm{THC}$, it seems unlikely that this particular chalcone possesses this mechanism of action. Other chalcones have also been reported to modulate nitric oxide production, or iNOS expression following LPS stimulation of a mouse macrophage cell line [36], the BV2 microglial cell line [27], and a rat microglial cell line [37] within similar compound concentration ranges of $1-50 \mu \mathrm{M}$. The exact signalling pathways inhibited by different chalcones may be diverse, as some studies suggest modulation of nuclear factor- $\kappa \mathrm{B}$, MAPK, or c-Jun pathways and others suggest STAT1 pathways may be involved $[26,37]$. 


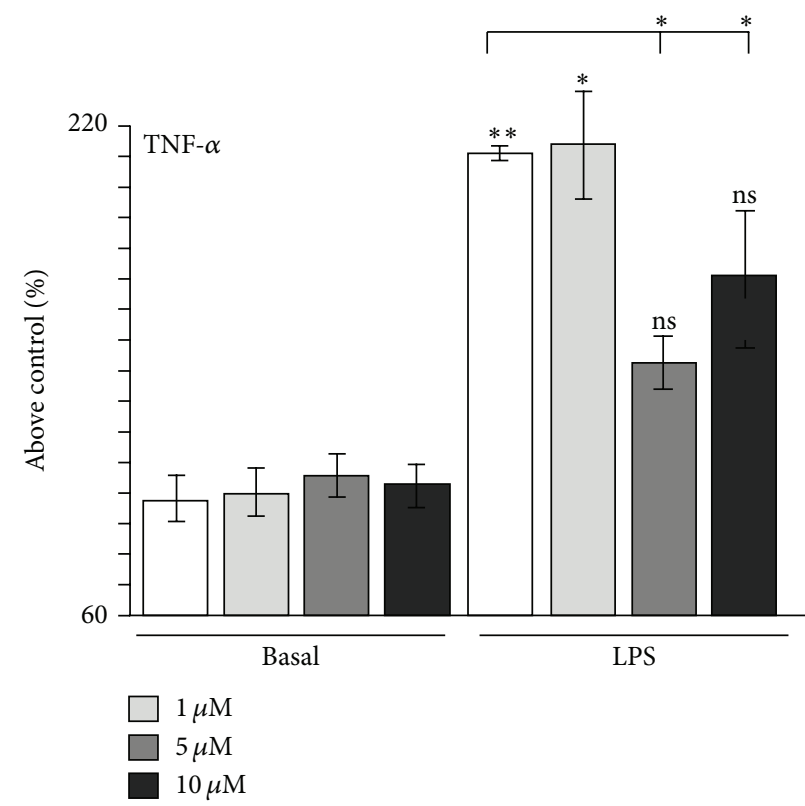

(a)

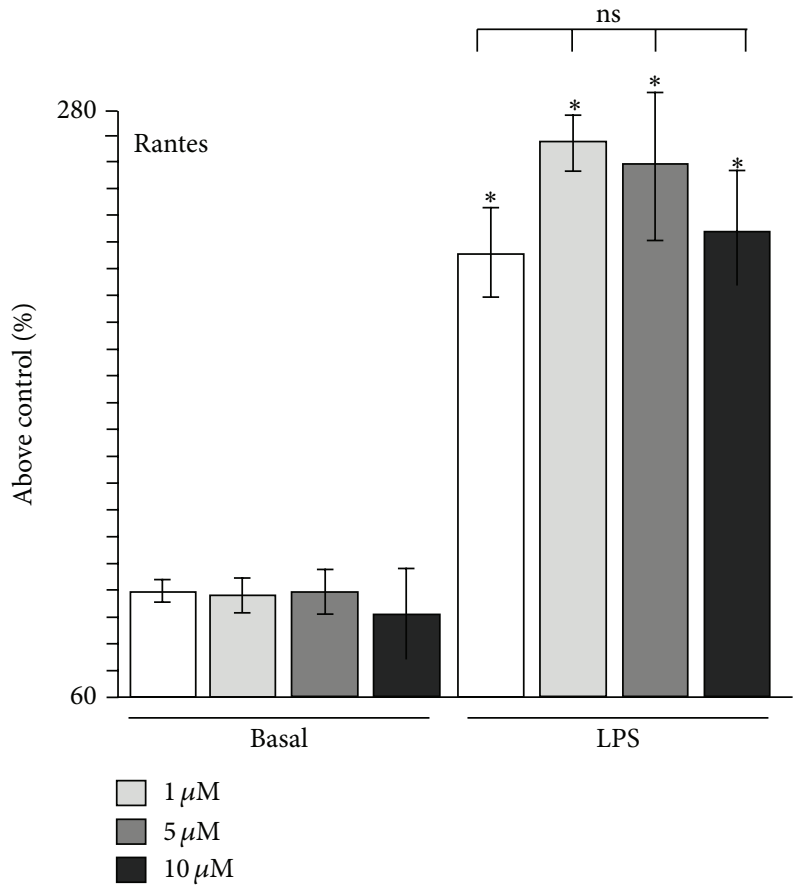

(c)

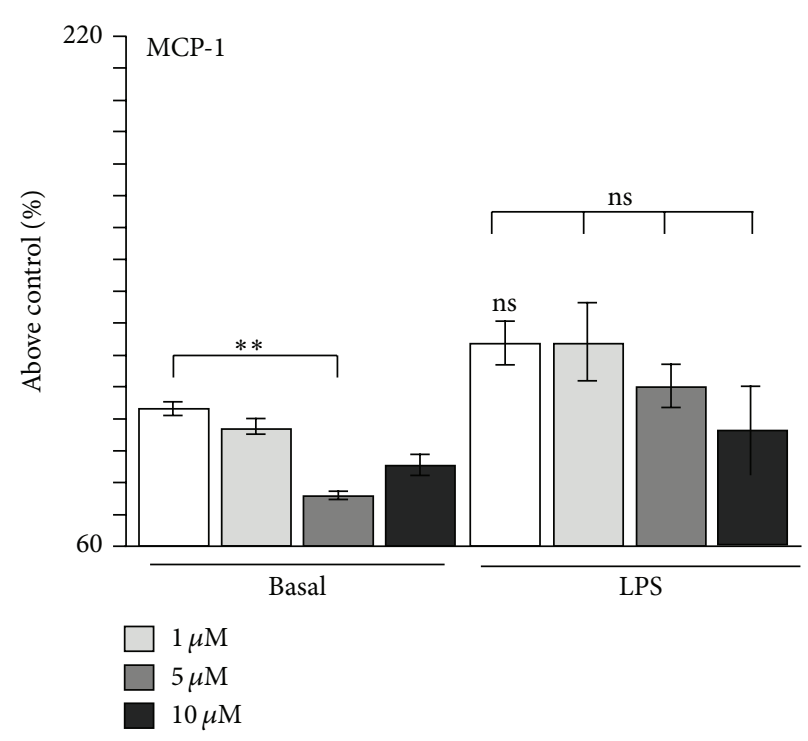

(b)

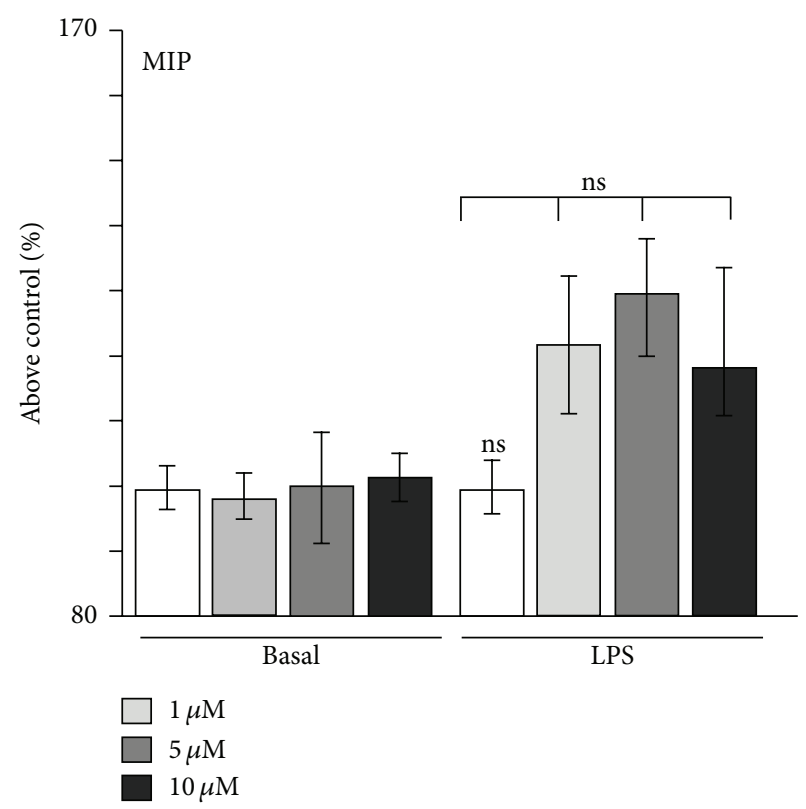

(d)

FIGURE 3: 225THC modulates inflammatory cytokine and chemokine responses in murine BV2 microglial cells. 225THC-modulation of cytokine/chemokine secretion by the BV2 microglial cell line. ELISA assessment of cytokine ((a) TNF- $\alpha$ ) and chemokine ((b) MCP-1; (c) RANTES; (d) MIP) secretion by BV2 microglial cells following stimulation with LPS $(2 \mu \mathrm{g} / \mathrm{mL})$ for $24 \mathrm{~h}$ in the presence of $225 T H C$ at the concentrations indicated. Statistical analysis was performed by a two-way ANOVA with Bonferroni post hoc analysis; $*$ indicates $p<0.05$, ${ }^{* *} p<0.01 ;$ ns, not significant when compared with basal or as indicated.

3.5. Modulation of Microglial Inflammatory Phenotype. Upon LPS stimulation, microglia adopt an inflammatory phenotype which is characterised by enhanced cell surface expression of key adhesion (CD11b, CD54), and costimulatory molecules (CD40). We investigated the effects of 225THC treatment on BV2 cell surface expression of CD11b, CD40, CD54, and CD68 in basal and LPS-treated BV2 microglia (Figure 5). Flow cytometric analysis (FACs) of marker mean fluorescence intensity (MFI) revealed no significant changes in basal CD40, CD54, CD68, or CD11b expression levels following 


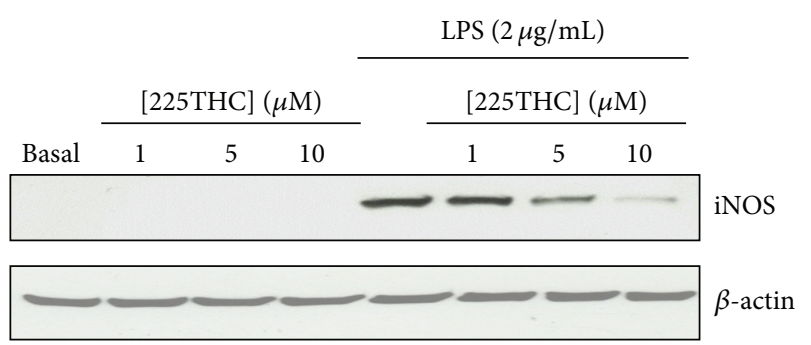

(a)

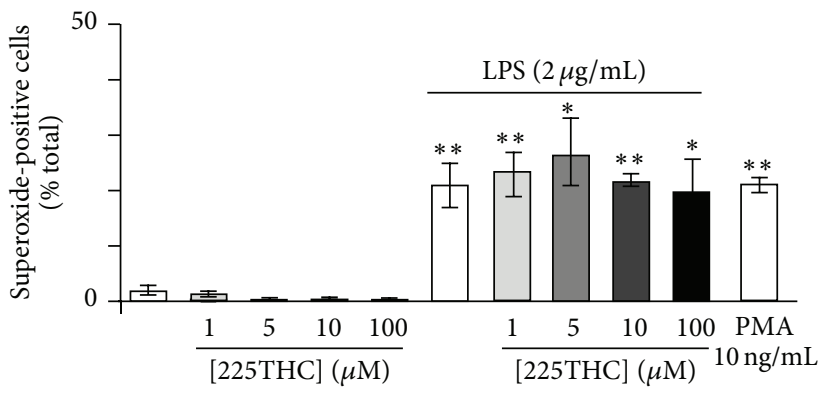

(c)

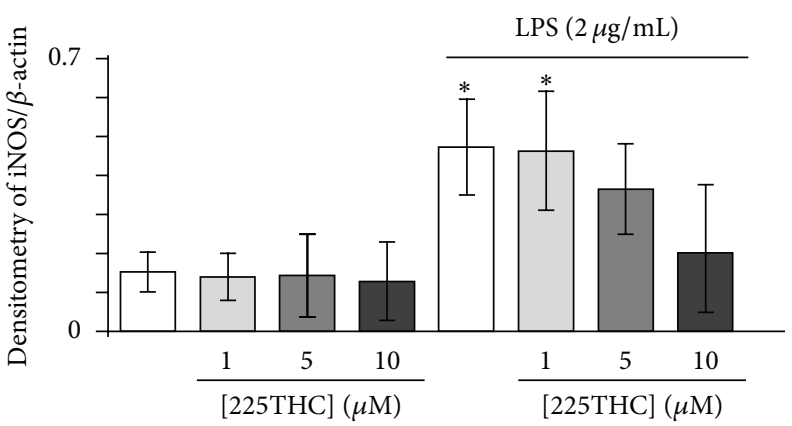

(b)
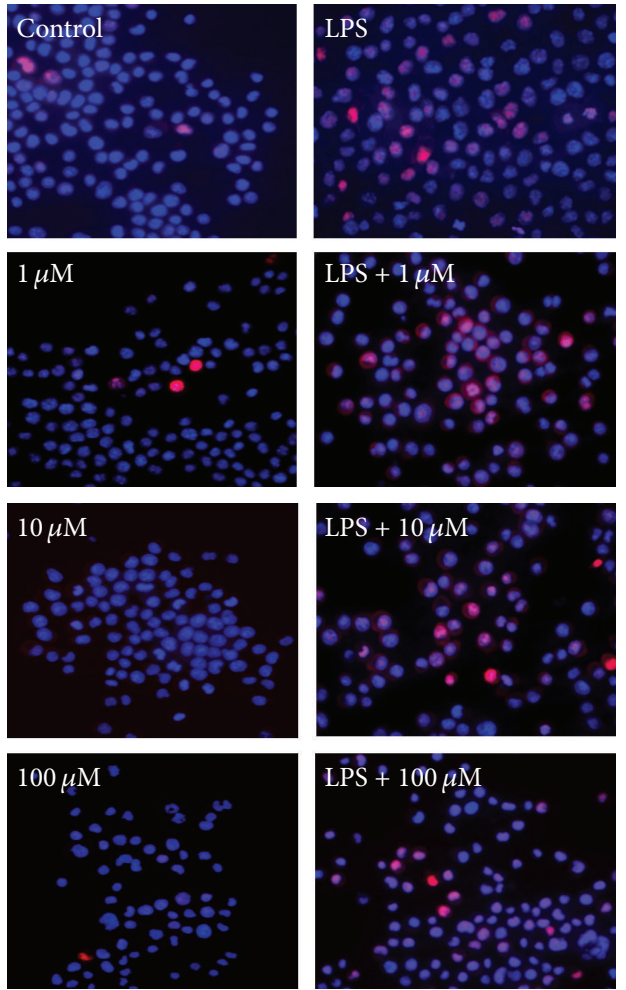

(d)

FIgURE 4: 225THC inhibits the LPS-induced expression of iNOS, but not NADPH-driven superoxide production, in BV2 microglia. Modulation of BV2 iNOS expression, but not superoxide production, by 225THC. Representative Western blots of iNOS expression and $\beta$ actin as a loading control (a) and densitometry (b) of three separate blots for BV2 microglia exposed to 225THC at the stated concentrations \pm LPS $(2 \mu \mathrm{g} / \mathrm{mL})$ for $24 \mathrm{~h}$. Statistical analysis was performed by a two-way ANOVA with Bonferroni post hoc analysis; $*$ indicates $p<0.05$ when compared with basal or as indicated. (c) Superoxide generation in BV2 microglia measured by dihydroethidium fluorescence following $24 \mathrm{~h}$ exposure to $225 \mathrm{THC}$ at the concentrations indicated \pm LPS $(2 \mu \mathrm{g} / \mathrm{mL})$. The phorbol, PMA, was used as a positive activator of superoxide generation. (d) Representative fields of BV2 microglia generating intracellular superoxide following $24 \mathrm{~h}$ exposure to $225 \mathrm{THC}+/-\mathrm{LPS}$. Statistical analysis was performed by a two-way ANOVA with Bonferroni post hoc analysis; $*$ indicates $p<0.05 ; * *$ indicates $p<0.01$ when compared with basal or as indicated.

$225 \mathrm{THC}$ treatment for $48 \mathrm{~h}$ (data not shown). However, following stimulation with $2 \mu \mathrm{g} / \mathrm{mL}$ LPS, CD40 (Figure 5(bii)), CD54 (Figure 5(cii)), and CD68 (Figure 5(dii)) were significantly upregulated and these changes in expression were abrogated in the presence of 5 or $10 \mu \mathrm{M}$ 225THC. CD40 appeared to be the most potently regulated marker, with both 5 and $10 \mu \mathrm{M}$ 225THC completely inhibiting LPS-induced upregulation (Figure 5(bii)) while the treatment had more moderate effects on CD54 and CD68 marker expression. As in the basal state, 225THC had no effect on CD11b expression by LPS-stimulated microglia (Figure 5(aii)).

Overall, these data suggest that 225THC targets the signalling pathways downstream of LPS binding and abrogates the expression of genes such as TNF- $\alpha$, iNOS, and CD40. Since these are all reported NF- $\kappa$ B driven genes, future work could investigate this further to identify the exact pathways modified by 225THC. Abrogating this gene expression produced a less neurotoxic microglial phenotype 


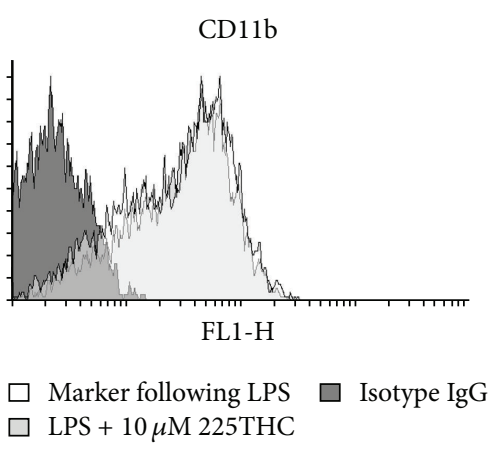

(ai)

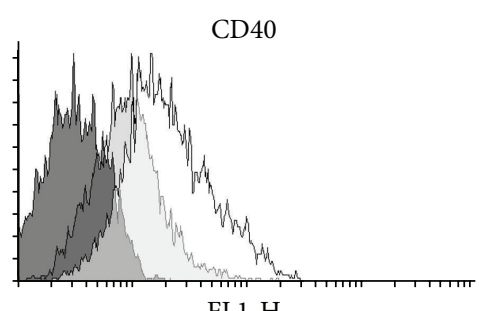

FL1-H

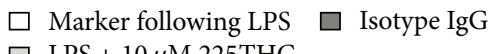
$\square$ LPS $+10 \mu \mathrm{M} 225 \mathrm{THC}$

(bi)

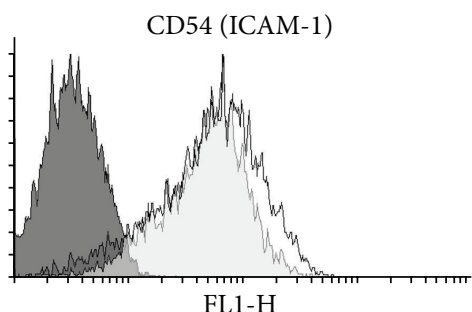

$\square$ Marker following LPS $\square$ Isotype IgG $\square$ LPS $+10 \mu \mathrm{M} 225 \mathrm{THC}$

(ci)

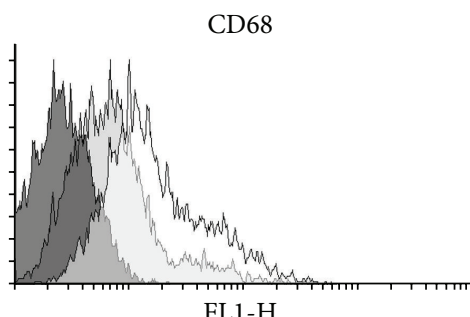

FL1-H

Marker following LPS $\square$ Isotype IgG $\square$ LPS $+10 \mu \mathrm{M} 225 \mathrm{THC}$

(di)

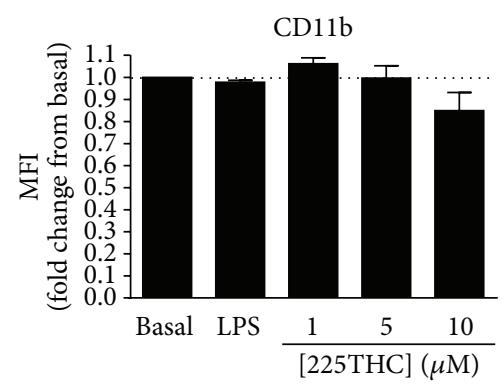

(aii)

(a)

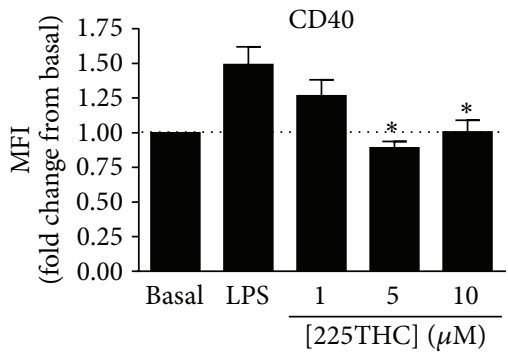

(bii)

(b)

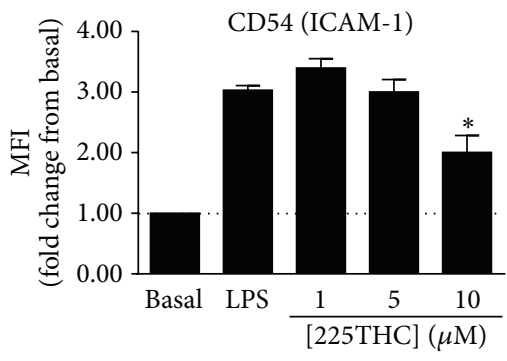

(cii)

(c)

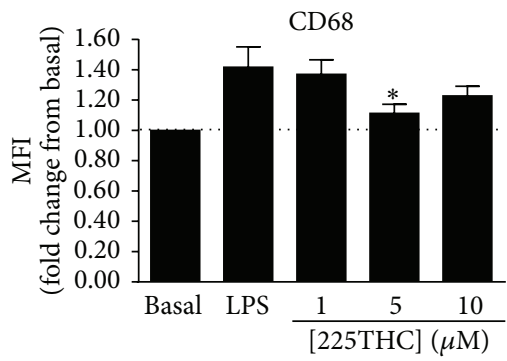

(dii)

(d)

FIGURE 5: 225THC treatment modulates inflammatory cell surface marker expression in LPS-treated BV2 microglia. Flow cytometric analysis of 225THC-treated BV2 microglia following LPS stimulation. (a) Representative histogram plots of BV2 cell surface CD11b, CD40, CD54, and CD68 expression following $2 \mu \mathrm{g} / \mathrm{mL}$ LPS-treatment for 48 hours (unfilled histograms), versus isotype control (black-filled histograms), and in the presence of $10 \mu \mathrm{M} 225 \mathrm{THC}$ (grey-filled histograms). (b) Summary data showing fold changes in CD11b, CD40, CD54, or CD68 expression by mean fluorescence intensity (MFI) from 4 independent experiments. Data are mean values \pm SEM where $*$ indicates $p<0.05$ by Student's $t$-test. 
which is likely to be beneficial for a number of neurodegenerative diseases in which aberrant microglial inflammatory reactivity is implicated. Future work could pinpoint further the microglial phenotype favoured by $225 \mathrm{THC}$ exposure and explore the genotype of the microglia.

\section{Abbreviations}

ANOVA: Analysis of variance

DPPH: 2,2-Diphenyl-1-picrylhydrazyl

dHEth: Dihydroethidium

CGCs: Cerebellar granule neurons

DIV: $\quad$ Days in vitro

DAPI: $\quad 4^{\prime}, 6$-Diamidino-2-phenylindole

DMSO: Dimethylsulphoxide

ECL: Electrochemiluminescence

ELISA: Enzyme-linked immunosorbent assay

$\mathrm{GABA}_{\mathrm{A}}: \quad \gamma$-Aminobutyric acid

IFN- $\gamma$ : Interferon- $\gamma$

IL- $1 \beta$ : Interleukin-1 $\beta$

IL-6: Interleukin-6

IL-10: Interleukin-10

iNOS: Inducible nitric oxide synthase

LPS: Lipopolysaccharide

MCP-1: CCL2/monocyte chemoattractant protein-1

MEM: $\quad$ Modified Eagle's medium

MIP: $\quad$ Macrophage inhibitory protein

MCP-1: Monocyte chemoattractant protein-1

NGS: $\quad$ Normal goat serum

PBS: $\quad$ Phosphate buffered saline

PFA: Paraformaldehyde

2-OH-E ${ }^{+}$: 2-Hydroxyethidium

RANTES: CCL5/regulation on activation normal T cell expressed and secreted

ROS: $\quad$ Reactive oxygen species

TNF- $\alpha$ : Tumour necrosis factor- $\alpha$.

\section{Conflict of Interests}

All authors declare no conflict of interests.

\section{Acknowledgments}

Manasi Jiwrajka would like to thank Dr. Kathleen Susman for her guidance. This research was supported by the University of Queensland Advantage Grant, Howard Hughes Medical Foundation Grant 52006322 to Vassar College, the Sherman Fairchild Foundation, and Vassar College and by general funds to the Pocock lab.

\section{References}

[1] S. Ducki, R. Forrest, J. A. Hadfield et al., "Potent antimitotic and cell growth inhibitory properties of substituted chalcones," Bioorganic and Medicinal Chemistry Letters, vol. 8, no. 9, pp. 10511056, 1998.

[2] M. L. F. Ferreyra, S. P. Rius, and P. Casati, "Flavonoids: biosynthesis, biological functions, and biotechnological applications," Frontiers in Plant Science, vol. 3, article 222, 15 pages, 2012.
[3] G. E.-D. A. A. Abuo-Rahma, M. Abdel-Aziz, M. A. E. Mourad, and H. H. Farag, "Synthesis, anti-inflammatory activity and ulcerogenic liability of novel nitric oxide donating/chalcone hybrids," Bioorganic and Medicinal Chemistry, vol. 20, no. 1, pp. 195-206, 2012.

[4] N. K. Sahu, S. S. Balbhadra, J. Choudhary, and D. V. Kohli, "Exploring pharmacological significance of chalcone scaffold," Current Medicinal Chemistry, vol. 19, no. 2, pp. 209-225, 2012.

[5] H. Wei, X. Zhang, G. Wu et al., "Chalcone derivatives from the fern Cyclosorus parasiticus and their anti-proliferative activity," Food and Chemical Toxicology, vol. 60, pp. 147-152, 2013.

[6] C. W. Mai, M. Yaeghoobi, N. Abd-Rahman, Y. B. Kang, and M. R. Pichika, "Chalcones with electron-withdrawing and electron-donating substituents: anticancer activity against TRAIL resistant cancer cells, structure-activity relationship analysis and regulation of apoptotic proteins," European Journal of Medicinal Chemistry, vol. 77, pp. 378-387, 2014.

[7] Z. Nowakowska, "A review of anti-infective and anti-inflammatory chalcones," European Journal of Medicinal Chemistry, vol. 42, no. 2, pp. 125-137, 2007.

[8] Y.-M. Lin, Y. Zhou, M. T. Flavin, L.-M. Zhou, W. Nie, and F.-C. Chen, "Chalcones and flavonoids as anti-tuberculosis agents," Bioorganic and Medicinal Chemistry, vol. 10, no. 8, pp. 27952802, 2002.

[9] J. Mojzis, L. Varinska, G. Mojzisova, I. Kostova, and L. Mirossay, "Anti-angiogenic effects of flavonoids and chalcones," Pharmacological Research, vol. 57, no. 4, pp. 259-265, 2008.

[10] F. Dal Piaz, A. Braca, M. A. Belisario, and N. De Tommasi, "Thioredoxin system modulation by plant and fungal secondary metabolites," Current Medicinal Chemistry, vol. 17, no. 5, pp. 479-494, 2010.

[11] N. Yadav, S. K. Dixit, A. Bhattacharya et al., "Antimalarial activity of newly synthesized chalcone derivatives in vitro," Chemical Biology and Drug Design, vol. 80, no. 2, pp. 340-347, 2012.

[12] F. V. DeFeudis, "Novel therapy for Alzheimer's disease," Neurobiology of Aging, vol. 10, no. 5, pp. 467-468, 1989.

[13] Y. Nomura and Y. Kitamura, "Inducible nitric oxide synthase in glial cells," Neuroscience Research, vol. 18, no. 2, pp. 103-107, 1993.

[14] P. J. Kingham, M. L. Cuzner, and J. M. Pocock, "Apoptotic pathways mobilized in microglia and neurones as a consequence of chromogranin A-induced microglial activation," Journal of Neurochemistry, vol. 73, no. 2, pp. 538-547, 1999.

[15] S. Singh, S. Swarnkar, P. Goswami, and C. Nath, "Astrocytes and microglia: responses to neuropathological conditions," International Journal of Neuroscience, vol. 121, no. 11, pp. 589-597, 2011.

[16] Y. Y. Deng, J. Lu, E.-A. Ling, and C. Kaur, "Role of microglia in the process of inflammation in the hypoxic developing brain," Frontiers in Bioscience (Scholar Edition), vol. 3, no. 3, pp. 884900, 2011.

[17] M. Dumont and M. F. Beal, "Neuroprotective strategies involving ROS in Alzheimer disease," Free Radical Biology and Medicine, vol. 51, no. 5, pp. 1014-1026, 2011.

[18] A. D. Kraft and G. Jean Harry, "Features of microglia and neuroinflammation relevant to environmental exposure and neurotoxicity," International Journal of Environmental Research and Public Health, vol. 8, no. 7, pp. 2980-3018, 2011.

[19] S. Cho, S. Kim, Z. Jin et al., "Isoliquiritigenin, a chalcone compound, is a positive allosteric modulator of GABA A receptors and shows hypnotic effects," Biochemical and Biophysical Research Communications, vol. 413, no. 4, pp. 637-642, 2011. 
[20] A. Elmann, A. Telerman, H. Erlank et al., "Protective and antioxidant effects of a chalconoid from Pulicaria incisa on brain astrocytes," Oxidative Medicine and Cellular Longevity, vol. 2013, Article ID 694398, 10 pages, 2013.

[21] M. Rossi, F. Caruso, E. J. Crespi et al., "Probing antioxidant activity of 2 '-hydroxychalcones: crystal and molecular structures, in vitro antiproliferative studies and in vivo effects on glucose regulation," Biochimie, vol. 95, no. 10, pp. 1954-1963, 2013.

[22] E. L. Mead, A. Mosley, S. Eaton, L. Dobson, S. J. Heales, and J. M. Pocock, "Microglial neurotransmitter receptors trigger superoxide production in microglia; consequences for microglialneuronal interactions," Journal of Neurochemistry, vol. 121, no. 2, pp. 287-301, 2012.

[23] C. M. Davenport, I. G. Sevastou, C. Hooper, and J. M. Pocock, "Inhibiting p53 pathways in microglia attenuates microglialevoked neurotoxicity following exposure to Alzheimer peptides," Journal of Neurochemistry, vol. 112, no. 2, pp. 552-563, 2010.

[24] N. C. Garbett, N. B. Hammond, and D. E. Graves, "Influence of the amino substituents in the interaction of ethidium bromide with DNA," Biophysical Journal, vol. 87, no. 6, pp. 3974-3981, 2004.

[25] A. Karlsson, J. B. Nixon, and L. C. McPhail, "Phorbol myristate acetate induces neutrophil NADPH-oxidase activity by two separate signal transduction pathways; dependent or independent of phosphatidylinositol 3-kinase," Journal of Leukocyte Biology, vol. 67, no. 3, pp. 396-404, 2000.

[26] F. Herencia, M. L. Ferrandiz, A. Ubeda et al., "4-Dimethylamino- $3^{\prime}, 4^{\prime}$-dimethoxychalcone down-regulates iNOS expression and exerts anti-inflammatory effects," Free Radical Biology \& Medicine, vol. 30, no. 1, pp. 43-50, 2001.

[27] N. Mateeva, M. Gangapuram, E. Mazzio, S. Eyunni, K. F. Soliman, and K. K. Redda, "Biological evaluation of synthetic chalcone and flavone derivatives as anti-inflammatory agents," Medicinal Chemistry Research, vol. 24, no. 4, pp. 1672-1680, 2015.

[28] S. C. Morgan, D. L. Taylor, and J. M. Pocock, "Microglia release activators of neuronal proliferation mediated by activation of mitogen-activated protein kinase, phosphatidylinositol-3kinase/Akt and delta-Notch signalling cascades," Journal of Neurochemistry, vol. 90, no. 1, pp. 89-101, 2004.

[29] J. Jebelli, T. Piers, and J. Pocock, "Selective depletion of microglia from cerebellar granule cell cultures using L-leucine methyl ester," The Journal of Visualized Experiments, no. 101, Article ID e52983, 2015.

[30] R. Von Bernhardi, J. E. Tichauer, and J. Eugenín, "Agingdependent changes of microglial cells and their relevance for neurodegenerative disorders," Journal of Neurochemistry, vol. 112, no. 5, pp. 1099-1114, 2010.

[31] C. Schwab and P. L. McGeer, "Inflammatory aspects of Alzheimer disease and other neurodegenerative disorders," Journal of Alzheimer's Disease, vol. 13, no. 4, pp. 359-369, 2008.

[32] A. Henn, S. Lund, M. Hedtjärn, A. Schrattenholz, P. Pörzgen, and M. Leist, "The suitability of BV2 cells as alternative model system for primary microglia cultures or for animal experiments examining brain inflammation," Altex, vol. 26, no. 2, pp. 83-94, 2009.

[33] S. Bano, K. Javed, S. Ahmad et al., "Synthesis of some novel chalcones, flavanones and flavones and evaluation of their antiinflammatory activity," European Journal of Medicinal Chemistry, vol. 65, pp. 51-59, 2013.
[34] M. Erta, A. Quintana, and J. Hidalgo, "Interleukin-6, a major cytokine in the central nervous system," International Journal of Biological Sciences, vol. 8, no. 9, pp. 1254-1266, 2012.

[35] S. L. Deshmane, S. Kremlev, S. Amini, and B. E. Sawaya, "Monocyte chemoattractant protein-1 (MCP-1): an overview," Journal of Interferon and Cytokine Research, vol. 29, no. 6, pp. 313-326, 2009.

[36] J. Rojas, J. N. Domínguez, J. E. Charris, G. Lobo, M. Payá, and M. L. Ferrándiz, "Synthesis and inhibitory activity of dimethylamino-chalcone derivatives on the induction of nitric oxide synthase," European Journal of Medicinal Chemistry, vol. 37, no. 8, pp. 699-705, 2002.

[37] H. Hara, Y. Nakamura, M. Ninomiya et al., "Inhibitory effects of chalcone glycosides isolated from Brassica rapa L. 'hidabeni' and their synthetic derivatives on LPS-induced NO production in microglia," Bioorganic \& Medicinal Chemistry, vol. 19, no. 18, pp. 5559-5568, 2011. 


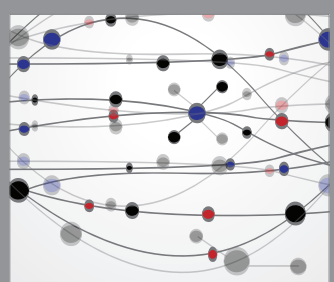

The Scientific World Journal
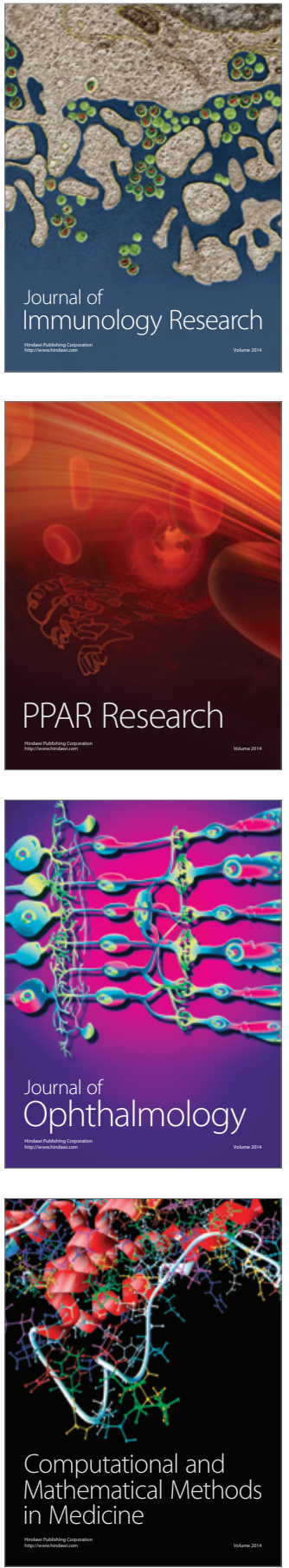

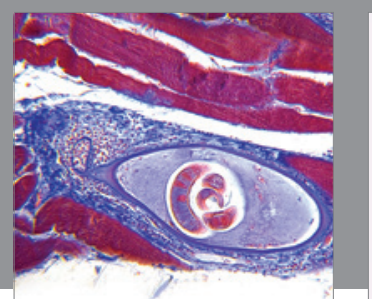

Gastroenterology Research and Practice

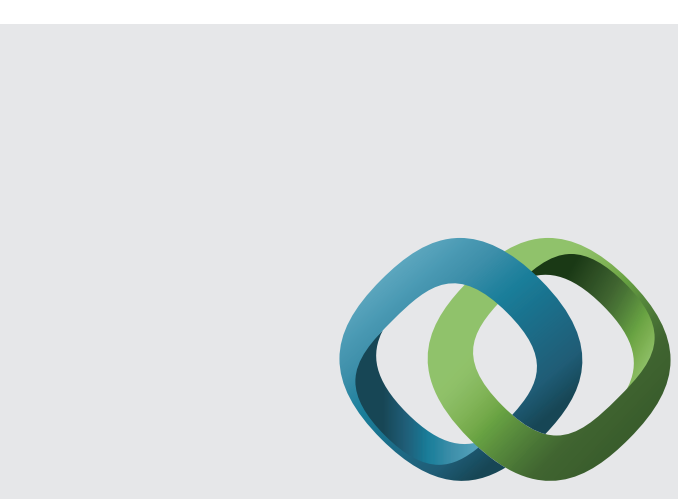

\section{Hindawi}

Submit your manuscripts at

http://www.hindawi.com
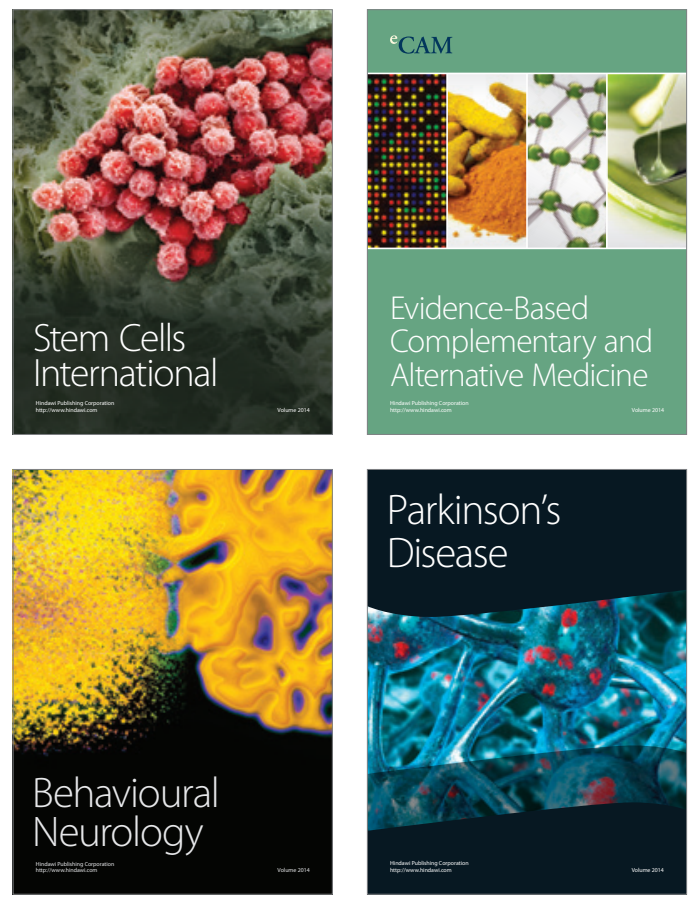
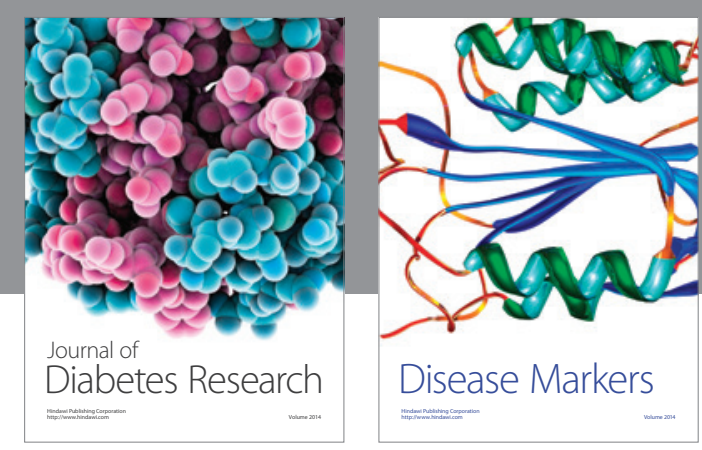

Disease Markers
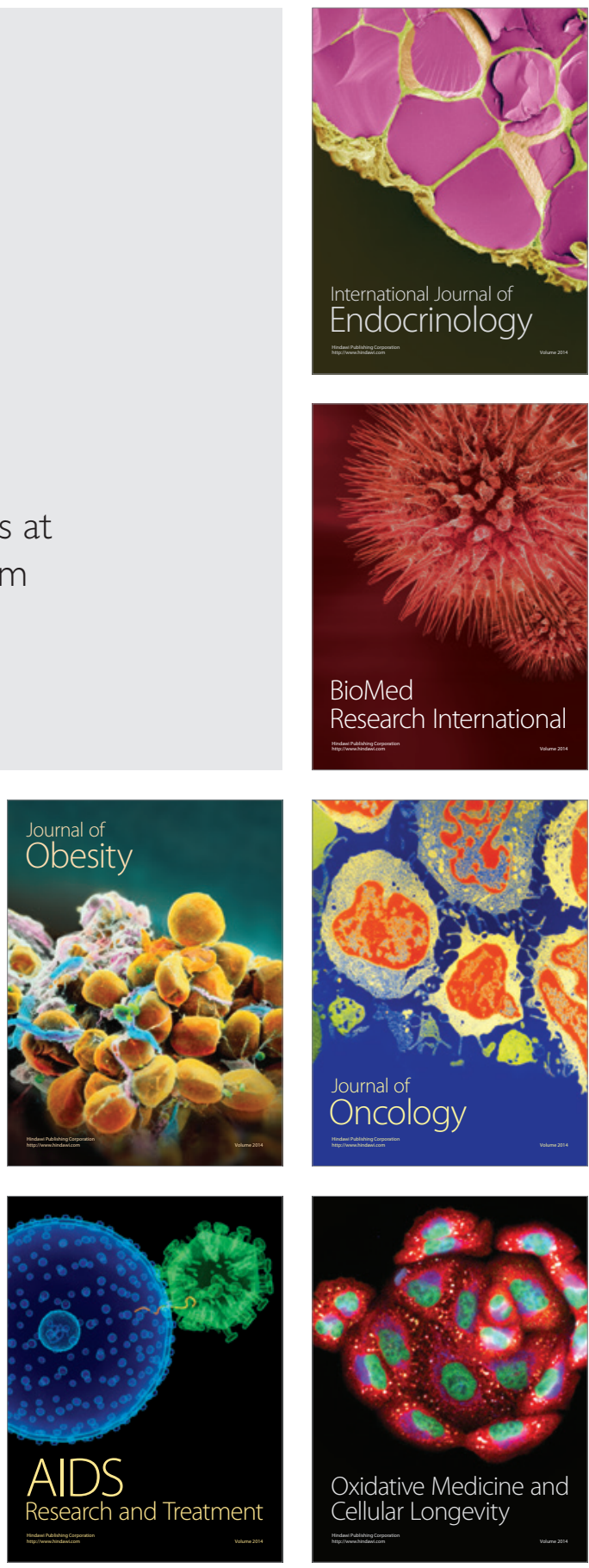